\title{
Malignant hyperthermia in dogs during general anaesthesia
}

\author{
Özlem Güzel1*, Defne Şadalak McKinstry²
}

\section{Review Article}

Volume: 1, Issue: 3

August 2017

Pages: 57-62

\section{Article History}

Received: 24.07 .2017

Accepted: 23.09.2017

Available online: 25.09.2017
1. Istanbul University, Veterinary Faculty, Surgery Department, Avcilar, Istanbul, Turkey. 2. Veterinary Surgeon, Bath, England.

\begin{abstract}
Malignant hyperthermia is a pharmacogenetic disorder of skeletal muscles developing as a hypermetabolic response to inhalation anaesthetics such as halothane, sevoflurane or isoflurane and depolarizing muscle relaxants such as succinylcholine. It is produced by mutation of the RYR1 gene in dogs. In anaesthetised dogs, regardless of the anaesthetic drugs used, calcium ion channel activity may change and malignant hyperthermia may develop. Clinical features are hyperthermia, tachypne, hypercarbia, increased oxygen consumption, severe metabolic acidosis, hyperkalemia, cardiac arrhytmias and muscle rigidity. For a diagnosis of malignant hyperthermia to be made, at least 3 of the clinical findings must be determined. Dantrolene sodium is specific antagonist of malignant hyperthermia. However, it is not usually preferred by veterinary practitioners due to its high cost as well as the low incidence of the occurrence of this complication. Therefore it is useful for alternative drugs such as acepromazine, diazepam and alfentanil to be used for the treatment of clinical findings manifested during malignant hyperthermia. The purpose of this review is to share with colleagues the latest information regarding the treatment of canine malignant hyperthermia occurring in relation to general anaesthesia
\end{abstract}

Keywords: hyperthermia, anaesthesia, dog.

DOI: 10.30704/http-www-jivs-net.330592

To cite this article: Güzel, Ö., \& Şadalak McKinstry, D. (2017). Malignant hyperthermia in dogs during general anaesthesia. Journal of Istanbul Veterinary Sciences, 1(3), 57-62. Abbreviated Title: J Ist Vet Sci

\section{Introduction}

Complications such as hypotension, hypothermia, respiratory and cardiac arrest are frequently encountered during general anaesthesia. Malignant Hyperthermia ( $\mathrm{MH}$ ) is a significant complication arising during general anaesthesia, however, not much is known about it particularly in the veterinary field.

$\mathrm{MH}$ is a pharmacogenetic disorder of skeletal muscles developing as a hypermetabolic response to inhalation anaesthetics such as halothane, sevoflurane or isoflurane and depolarizing muscle relaxants such as succinylcholine. Currently, it is not considered to be a disease but rather a clinical syndrome. This condition is termed Malignant Hyperthermia Syndrome (MHS) and stems from pathophysiological changes as well as multiple genetic and environmental factors. Compared to other inhalation anaesthetics, the gas with the

* Corresponding Author: Özlem Güzel, Istanbul University, Faculty of Veterinary Medicine, Department of Surgery, Istanbul, Turkey, E-mail: drozlemguzel@gmail.com highest trigger potential is halothane (Brunson and Hogan, 2004; Steffey and Mama, 2007; Adami et al., 2012).

The occurrence rate in humans is between 1:5000 to 1:50000-100000. While widespread in pigs, sporadic cases have been reported in dogs, cats and horses (Haskins, 2007; Schneiderbanger et al., 2014). The incidence in dogs remains unknown. It has been stated that it is seen in $1: 2000$ or $1: 4000$ of veterinary anaesthesia cases and leads to death and various complications (O'Brien et al., 1983). MH may be seen in different breed dogs such as Greyhounds, Border Collies, Cocker Spaniels, Doberman Pinchers, Pointers, Saint Bernards and Kangal Shepherd dogs. However, all dogs carry a potential risk (O'Brien et al., 1983; Chohan and Greene, 2011; Guzel et al., 2016). 


\section{The Reasons of Malignant Hyperthermia}

Pathophysiological changes occurring in MHS originate from the uncontrolled release of intracellular calcium $(\mathrm{Ca}++)$ from the sarcoplasmic reticulum of skeletal muscles into the myoplasm, subjected to halogenised volatile anaesthetics and depolarizing muscle relaxants (Kirmayer, et al., 1984; Brunson and Hogan, 2004; Kim, 2012). In individuals sensitive to malignant hyperthermia, trauma, surgical interventions, physical activity, transportation and other causes of stress factors may trigger this syndrome in the absence of anaesthesia. Attacks may also be observed during the anaesthesia recovery period (Gronert and Milde, 1981; Kirmayer, et al., 1984). It is produced by mutation of the RYR1 gene in dogs (Roberts et al., 2001; Brunson and Hogan, 2004). In anaesthetized dogs, regardless of the anaesthetic drugs used, calcium ion channel activity may change and $\mathrm{MH}$ may develop (Lemke, 2007). Molecular genetic tests are used in determining gene mutation for MHS. However, at present these tests are available only in Europe and North America (Chohan and Greene, 2011; Kim, 2012).

\section{Clinical Findings of Malignant Hyperthermia}

Classic findings of $\mathrm{MH}$ are those relating to hypermetabolic reactions of the body such as; rapid increase in body temperature, tachypnea, hypercarbia, increased oxygen consumption, severe metabolic acidosis, hyperkalaemia, cardiac arrhythmias and rigidity in skeletal muscles (O'Brien et al., 1983; Adami et al., 2012). Severe hyperthermia causes multiple organ failure in the renal, hepatic and gastrointestinal systems, heart muscle damage, muscle necrosis, cerebral oedema, widespread intravascular coagulation, hypoxaemia, metabolic acidosis and hyperkalaemia (Kim, 2012; Schneiderbanger et al., 2014; Rosenberg et al., 2015).

When comparing to humans and pigs, the metabolic acidosis developed in dogs is mild and muscle rigidity is less (Nelson, 1991; Roberts et al., 2001; Brunson and Hogan, 2004). While muscle rigidity may be observed in all muscles, it may also be restricted to the masseter muscles and trismus occurs (O'Brain et al., 1983; Kirmayer et al., 1984; Krause et al., 2004). The earliest finding of $\mathrm{MH}$ in dogs is an increase in carbondioxide (CO2). While end tidal CO2 (ETCO2) increases in cases given automatic ventilation, hyperventilation is observed in patients with spontaneous respiration. $\mathrm{CO} 2$ increase begins within 10-14 minutes of commencing halothane anaesthesia (Nelson, 1991; Krause et al., 2004; Adami et al., 2012). Secondly, increase in rectal temperature must be assessed. Rectal temperature displays an increase within 10-14 minutes following halothane or isoflurane anaesthesia (Nelson, 1991; Adami et al., 2012). However, it may develop during any stage of anaestheia as well as in the early post-operative period. Body temperature may increase by 1 oC every 5 minutes and reach 43oC (Kirmayer et al., 1984; Rosenberg et al., 2015).

\section{Diagnosing Malignant Hyperthermia}

The majority of malignant hyperthermia cases consist of healthy animals with no history of anaesthesia problems and showing no prior warning signs. Therefore, patients to be anaesthetised are investigated regarding the presence of any littermates, dam, sire or offspring with unexplained death due to anaesthesia (Kim, 2012; Rosenberg et al., 2015). For a diagnosis of malignant hyperthermia to be made, at least 3 of the following findings must be determined: cardiac arrhythmias, acidosis, hypercarbia, raised body temperature and muscle rigidity. A single finding on its own is not specific for the disease (Brunson and Hogan, 2004; Chohan and Greene, 2011). A persistent rise in the carbondioxide level and body temperature despite extreme respiratory support during anaesthesia is considered to be positive MH until proven otherwise (Brunson and Hogan, 2004). Laboratory findings reveal increased serum glucose, phosphorus, magnesium, potassium, creatine kinase and alkaline phosphatase levels. Subsequently, calcium and potassium levels decrease (Kirmayer et al., 1984; Otto, 1992).

\section{Treatment}

Cessation of halogenated anaesthetic gases or depolarizing muscle relaxants forms the most important step of the treatment. The anaesthesia machine is flushed with $100 \%$ oxygen in order to remove residual anaesthetics. To complete surgery, anaesthesia is continued with opioids, sedatives and non-depolarising muscle relaxants (Kirmayer et al., 1984; Nelson, 1991; Krause et al., 2004). Intravenous fluid administration is started and blood samples are taken for laboratory analysis. Since the reason of death in these patients is rhythm disorders due to hyperkalaemia, the patient is hyperventilated with $100 \% 02$. If metabolic acidosis has 
developed or is suspected to develop, sodium bicarbonate is administered at a dose of $1-2 \mathrm{mEq} / \mathrm{kg} \mathrm{IV}$. In addition to sodium bicarbonate, administration of crystalised insulin (0.25-0.5) in 50\% dextrose $(0.5 \mathrm{ml} /$ $\mathrm{kg}$ ) enables the potassium to enter muscle and other depot cells. In cases where malignant hyperthermia occurs together with hyperkalaemia, it is not recommended to use calcium chloride and calcium gluconate (Nelson, 1991; Krause et al., 2004; Rosenberg et al., 2015).

The specific antagonist of pathophysiological changes caused by $\mathrm{MH}$ is dantrolene sodium (Brunson and Hogan, 2004; Haskins, 2007; Adami et al., 2012). Dantrolene sodium is a ryanodine receptor antagonist. It prevents excessive calcium release from the sarcoplasmic reticulum of skeletal muscles in affected individuals, therefore inhibiting excitation-contraction coupling (Saltzman et al., 1984; Lynch et al., 1986; Fruen et al., 1997).

Use of dantrolene $(2.5-10 \mathrm{mg} / \mathrm{kg}$ IV) for treatment is life-saving for this disease (Fruen et al., 1997; Adami et al., 2012; Guzel et al., 2016). The drug should be given as fast as possible in a single dose ( $3 \mathrm{mg} / \mathrm{kg}$ IV). The drug may be used every 5 minutes until the clinical findings of hypermetabolism are eliminated and the total dose reaches $10 \mathrm{mg} / \mathrm{kg}$ (Nelson, 1991; Krause et al., 2004; Chohan and Greene, 2011).

Dantrolene has high solubility in oil, while low solubility in water. It is available as lyophilized powder (20 mg) with added mannitol (3 gr) to increase its solubility. Dantrolene has a pH value of 9.5 and is a highdegree alkaline. Therefore, it should be diluted with 60 ml of water before use (Karan et al., 1996; Schneiderbanger et al., 2014). Heating the solution to $40 \mathrm{oC}$ beforehand enables the powder to dissolve. Since dantrolene is an irritant for veins, it must be administered as a rapid intravenous infusion using a larger cannula (Krause et al., 2004).

When dantrolene is used, calcium entry blockers (verapamil) must not be given (Kirmayer et al., 1984; Rosenberg et al., 2015). Simultaneous administration of these drugs significantly decreases cardiac function (Saltzman et al., 1984; Lynch et al., 1986).

Using room temperature fluids to cool the surface is an effective method in cooling down the patient. Water evaporation from the skin surface allows the body to cool down. Using icy water causes vasoconstriction. This delays the drop in body temperature until skin temperature has dropped by $10 \mathrm{oC}$. From this point, the blood vessels become paralysed, vasodilatation takes place and body temperature decreases rapidly (Krause et al., 2004; Haskins, 2007).

However, cooling of the patient is controversial. The cooling process causes vasoconstriction and this, in turn, may delay the entry of dantrolene into skeletal muscles. Use of cooled intravenous fluids may be beneficial to cool down the patient. In the case of $\mathrm{MH}$ developed during a surgical intervention in a body cavity, lavage of the abdomen and thorax may be performed using icy water. However, priority must be given to completing the surgical intervention. The cooling process is continued until body temperature has reached $38{ }^{\circ} \mathrm{C}$. It is important that the body temperature does not drop lower than $38{ }^{\circ} \mathrm{C}$. This would make it more difficult to manage dysrhythmia and coagulopathy (Kirmayer et al., 1984; Schneiderbanger et al., 2014).

Fans and ice packs may be used as a supplementary method in decreasing body temperature. Administering large volumes of crystalloid solutions to the colon, stomach or body cavities provides an effective method of internal cooling. Use of antipyretic drugs (antiprostaglandines, dipyrone, aminopyrone) may be effective in lowering fever, however, it is ineffective in treating pathologic hyperthermia (Haskins, 2007; Kim, 2012; Chohan and Greene, 2011).

Use of non-depolarising muscle relaxants in the acute stage is not effective as a method of treatment. However, these prevent muscle depolarization and contraction by blocking the receptors to which acetylcholine binds in the neuromuscular junction. In the absence of dantrolene, $\mathrm{MH}$ reactions can be prevented using non-depolarising neuromuscular blockers (Gronert and Milde, 1981; Chohan and Greene, 2011).

In advanced $\mathrm{MH}$ cases, aggressive fluid replacement and volume expansion must be carried out to prevent the occurrence of hypovolaemia and hypotension. Saline solution and $5 \%$ dextrose can be used for this. However, calcium added Lactated Ringer solution is not recommended. Shock treatment volumes $(90 \mathrm{ml} / \mathrm{kg} /$ hour, IV) may be required for blood pressure and kidney perfusion (Kirmayer et al., 1984; Brunson and Hogan, 2004).

\section{Monitoring Patients After Anaesthesia}

Patients must be closely monitored in the postanaesthesia stage. Monitoring blood pressure and 
performing blood gas analysis aids in determining the fluid amount to be administered both for volume support and to correct metabolic acidosis. Blood samples are taken to measure blood gases, acid-base balance, creatinine (CK), serum electrolyte values, serum lactate and blood glucose concentration (Krause et al., 2004; Schneiderbanger et al., 2014).

ECG monitoring must be performed (Kirmayer et al., 1984; Brunson and Hogan, 2004). Premature ventricular contractions (PVCs) and ventricular tachycardia may be prevented by administering boluses of lidocaine or procainamide $(1-2 \mathrm{mg} / \mathrm{kg})$. For the treatment of permanent arrhythmias, recurrent doses or continuous infusions may be required (Kim, 2012). Some researchers (Kirmayer et al., 1984; Chohan and Greene, 2011) state that use of lidocaine in the treatment of tachyarrhythmias may increase myoplasmic calcium, worsening the hyperthermic situation, therefore, it is more beneficial to use procainamide instead. In patients that survive from the acute hyperkalaemia phase, renal failure due to rhabdomyolysis develops. Death follows as a result of extensive intravascular coagulopathy. For this reason, patients must be catheterized, urine should be collected and myoglobin levels should be checked. Osmotic and loop diuretics must be used to prevent high densities of myoglobin and protein causing renal failure (Rosenberg et al., 2015).

In the event of halting $\mathrm{MH}$ at the start, there is no recurrence 24-48 hours after the first signs of the condition developing. Full recovery may be expected with suitable treatment (Brunson and Hogan, 2004).

\section{Alternative Treatment Methods in Malignant Hyperthermia}

Dantrolene, the life-saving drug for $\mathrm{MH}$, is not usually present in veterinary practices due to its high cost as well as the low incidence of the occurence. Therefore, it is useful for alternative drugs to be used for the treatment of clinical findings manifested during $\mathrm{MH}$. One of these drugs is acepromazine, which is recommended for lowering body temperature. By way of decreasing the catecholamine level in the thermoregulatory centre of the hypothalamus and at the same time blocking peripheral alpha 1 adrenergic receptors, acepromazine generates vasodilatation and hypothermia. It also produces muscle relaxation without providing analgesia (Lemke, 2007; Adami, 2012).

Another drug is diazepam, the most frequently used benzodiazepine in veterinary medicine. It produces a good level of muscle relaxation and has an anticonvulsant effect. It is therefore effective in treating muscle convulsions developed during $\mathrm{MH}$. The vasodilatation produced by the drug is effective in lowering body temperature (Lemke, 2007; Adami et al., 2012; Guzel et al., 2016).

Alfentanil contributes to lowering body temperature by producing vasodilatation and decreasing raised heart rate (HR) and respiratory rate (RR) and can be added to the treatment. This procedure decreases the HR and RR to normal ranges in $\mathrm{MH}$ (Lamont and Mathews, 2007; Guzel et al., 2016). Also alfentanil administration will decrease pain due to surgery, alleviate stress and therefore, may prevent triggering of hyperthermia (Brunson and Hogan, 2004).

\section{Safe Anaesthesia in Malignant Hyperthermia}

When dogs with malignant hyperthermia or those suspected of developing it require anaesthesia, volatile anaesthetics such as halothane, isoflurane and sevoflurane and depolarizing neuromuscular blocking agents such as succinylcholine should not be used. Benzodiazepines, phenothiasines, barbiturates, etomidate, propofol, dissociative drugs, opioids, nitrous oxide, non-depolarising neuromuscular blockers and local anaesthetics may be used safely (Brunson and Hogan, 2004; Rosenberg et al., 2015).

To minimize pre-anaesthesia stress, patients should be given effective premedication. For this purpose, drugs with a sedative effect such as diazepam, midazolam and acepromazine are recommended.

Opioids are used to establish effective analgesia. Although there is no specific research, alpha 2 agonist drugs such as xylazine, detomidine and medetomidine are also considered to be safe (Kirmayer et al., 1984; Brunson and Hogan, 2004).

Anaesthesia induction of patients is carried out with standard anaesthetics such as thiopental, propofol, etomidate, ketamine or tiletamine-zolazepam. Anaesthesia may be maintained with the same drugs. Drug doses are determined according to the doses recommended by general use or to the manufacturer's instruction. Since propofol is rapidly eliminated and 
does not accumulate following multiple administrations, it is suitable for repeat use (Brunson and Hogan, 2004; Chohan and Greene, 2011).

Due to their protective effect against $\mathrm{MH}$, acetylpromazine and thiopental administration for premedication and induction may prevent these findings occurring in the first 15 minutes of halothane anaesthesia (Gronert and Milde, 1981; O’Brien et al., 1983).

Local and regional anaesthesia techniques may also be used in patients with MH. However, the stress caused by physical restrictions carried out in such procedures may initiate $\mathrm{MH}$ reactions. Therefore, it is important to perform sufficient sedation before local or regional anaesthesia procedures (Rosenberg et al., 2015).

Respiratory support and oxygen reinforcement must be carried out in $\mathrm{MH}$ positive patients or those

\section{References}

Adami, C., Axiak, S., Raith, K., \& Spadavecchia, C. (2012). Unusual perianesthetic malignant hyperthermia in a dog. Journal of the American Veterinary Medical Association, 240(8), 894-453.

Brunson, D. B., \& Hogan, K. J. (2004). Malignant hyperthermia: a syndrome not a disease. The Veterinary Clinics of North America. Small Animal Practice, 34, 14191433.

Chohan, A. S., \& Greene, S. A. (2011). Anesthesia case of the month. Malignant hyperthermia. Journal of the American Veterinary Medical Association, $239\left({ }^{1}\right),{ }^{3} 7^{0}-940$.

Fruen, B. R., Mickelson, J. R., \& Louis, C. F. (1997). Dantrolene inhibition of sarcoplasmic reticulum $\mathrm{Ca} 2+$ release by direct and specific action at skeletal muscle ryanodine receptors. The Journal of Biological Chemistry, 272(68), 26965-26971.

Gronert, G. A., \& Milde, J. H. (1981). Variations in onset of porcine malignant hyperthermia. Anesthesia and Analgesia, $60\left(^{1}\right), 8^{3} 3-503$.

Guzel, O., Yildar, E., Karan, B., \& Aydin, D. (2016, May 11-14). Malignant hyperthermia induced general anesthesia in an Anatolian Shepherd Dog. Paper presented at the 5th International Turkey Veterinary Surgery Congres. Erzurum, Turkey.

Haskins, S. C. (2007). Monitoring anesthetized patients. In W.J. Tranquilli, J.C. Thurmon, K.A. Grimm (Ed). Lumb \& Jones' Veterinary Anesthesia and Analgesia. 4th ed. (pp. 533 - 558). Iowa, US : Blackwell Publishing.

Karan, S. M., Lojeski, E. W., Haynes, D. H., Bina, S., Wesche, D. L., Boedeker, B. H., \& Muldoon, S. M. (1996). Intravenous suspected. End tidal carbondioxide analysis must be performed to determine $\mathrm{CO} 2$ increase, which is the first sign of the $\mathrm{MH}$ reaction. It is essential to monitor cardiovascular parameters such as blood pressure, HR and ECG as well as body temperature (Nelson, 1991; Brunson and Hogan, 2004).

\section{Conclusion}

Malignant hyperthermia is a preventable clinical condition. By carrying out a few changes such as reducing physical activity that causes stress, and lowering surrounding temperature, affected dogs may continue their daily activity and normal life (Brunson and Hogan, 2004). The purpose of this review is to share with colleagues the current information regarding the treatment of canine $\mathrm{MH}$ occurring in relation to general anaesthesia and its clinical picture, in the hope that sufficient information will help in saving the life of animals.

lecithin-coated microcrystals of dantrolene are effective in the treatment of malignant hyperthermia: An investigation in rats, dogs, and swine. Anesthesia and Analgesia, 82, ${ }^{130}-802$.

Kim, D.C. (2012). Malignant hyperthermia. Korean Journal of Anesthesiology, 63 (5), 391-401.

Kirmayer, A. H., Klide, A. M., \& Purvance, J. E. (1984). Malignant hyperthermia in a dog: Case report and review of the syndrome. Journal of the American Veterinary Medical Association, 185(9), 978- 982.

Krause, T., Gerbershagen, M., Fiege, M., Weißhorn, R., \& Wappler, F. (2004). Dantrolene - A review of its pharmacology, therapeutic use and new developments. Anaesthesia, 59, 364-373.

Lamont, L. A., \& Mathews, K. A. (2007): Opioids, nonsteroidal anti-inflammatories, and analgesic adjuvants. In W. J. Tranquilli, J. C. Thurmon, K. A. Grimm (Ed). Lumb \& Jones' Veterinary Anesthesia and Analgesia. 4th ed. (pp. 241-271). Iowa, US: Blackwell Publishing.

Lemke, K. A. (2007). Anticholinergics and sedatives. In W.J. Tranquilli, J.C. Thurmon, K.A. Grimm (Ed). Lumb \& Jones' Veterinary Anesthesia and Analgesia. 4th ed. (pp. 203239). Iowa, US: Blackwell Publishing.

Lynch, C., Durbin, C. G., Fisher, N. A., Veselis, R. A., \& Althaus, J. S. (1986). Effects of dantrolene and verapamil on atrioventricular conduction and cardiovascular performance in dogs. Anesthesia and Analgesia, 21, 252258. 
Nelson, T. E. (1991). Malignant hyperthermia in dogs. Journal of the American Veterinary Medical Association, 198(6), 989 - 994.

O’Brien, P. J., Cribb, P. H., White, R. J., Olfert, E. D., \& Steiss, J. E. (1983). Canine malignant hyperthermia: Diagnosis of susceptibility in a breeding colony. The Canadian Veterinary Journal, 24, 51 6-177.

Otto, K. (1992). Malignant hyperthermia as a complication of anesthesia in the dog. Tierärztliche Praxis, 20(9), $95^{3}$ 522.

Roberts, M. C., Mickelson, J. R., Patterson, E. E., Nelson, T. E., Armstrong, P.J., Brunson, D.B., \& Hogan, K. (2001) Autosomal dominant canine malignant hyperthermia is caused by a mutation in the gene encoding the skeletal muscle calcium release channel (RYR1). Anesthesiology, $95,{ }^{1} 5^{0}-725$.
Rosenberg, H., Pollock, N., Schiemann, A., Bulger, T., \& Stowell, K. (2015). Malignant hyperthermia: a review. Orphanet Journal of Rare Diseases, $10\left({ }^{3} 7\right) .5-19$.

Saltzman, L. S., Kates, R. A., Corke, B. C., Norfleet, E. A., \& Heath, K. R. (1984). Hyperkalemia and cardiovascular collapse after verapamil and dantrolene administration in swine. Anesthesia and Analgesia, 63, 81 7-478.

Schneiderbanger, D., Johannsen, S., Rower, N., \& Schuster, F. (2014). Management of malignant hyperthermia: Diagnosis and treatment. Therapeutics and Clinical Risk Management, 10, 799-362.

Steffey, E. P., \& Mama, K. R. (2007): Inhalation anesthetics. In W.J. Tranquilli, J.C. Thurmon, K.A. Grimm (Ed). Lumb \& Jones' Veterinary Anesthesia and Analgesia. 4th ed. (pp. 355-394). Iowa, US: Blackwell Publishing. 